\title{
Determining the pressure modes at high frequencies in lined ducts with a shear flow
}

\author{
O. Olivieri, A. McAlpine, and R.J. Astley \\ Institute of Sound and Vibration Research, University of Southampton, SO17 1BJ, UK
}

\begin{abstract}
Noise transmission problems where sound propagates in flow ducts occur in a variety of contexts. The work in this paper is motivated by the turbofan aircraft engine application. Simulating the acoustics of turbofan duct systems is a challenging problem because this can necessitate the modelling of high-frequency sound propagation in ducts containing sheared mean flow and with acoustically-lined walls. The purpose of this work is to develop fast and efficient simulation methods, that can be used to calculate the propagation of sound in lined flow ducts. In this paper, preliminary results are presented obtained from a new finite element code which has been developed specifically to determine the pressure modes in ducts containing shear flow. The formulation of the finite element method is outlined. Preliminary results are shown which compare the duct modes with different boundary layer profiles. These results provide examples of finite element solutions of pressure modes in an annular duct. The results show how the shape of the boundary-layer profile can affect the duct modes.
\end{abstract}

\section{Introduction}

The adoption of state-of-the-art noise-reduction technologies is of primary interest in the aerospace industry. To achieve optimal fuel efficiency, modern turbofan aero-engines are designed with very high bypass ratios (up to 9:1), which results in fan noise being a dominant noise source at both take-off and landing. An effective means of reducing fan noise is through the design of sound-absorbing intake and bypass turbofan duct acoustic liners, which can be 'tuned' to an optimum frequency range, resulting in significant attenuation of part of the fan noise spectrum.

Earlier liner designs were based on prediction methods where the intake and bypass ducts were typically modelled as straight, axisymmetric, circular and annular ducts, containing uniform mean flow. These methods used the Ingard-Myers lined boundary condition ${ }^{1}$ formulated by imposing a vortex sheet at the duct walls. It has become evident that such models are not sufficiently accurate to foster the development of the next generation of acoustic liner configurations.

More accurate mathematical models, which take into account the complex geometry and flow field of a turbofan duct system are nowadays used more routinely. Sound transmission in more realistic intake and bypass duct geometries can be solved using numerical techniques; for example, finite element (FE) methods, based on solutions of the convected wave equation, are also commonly used in duct acoustics. These frequency domain methods use an irrotational mean flow, with the Myers lined boundary condition. However, these methods cannot be used with a parallel shear flow. In general, with a sheared mean flow, solutions of the linearized Euler equations (LEE) in the frequency domain can be obtained, but at high frequencies the problem size can become prohibitively large.

In this paper we investigate the pressure modes in ducts containing a mean sheared flow comprised of uniform core flow and boundary layers at the duct walls, and with acoustically-lined walls. A key objective is to accurately predict the sound attenuation in lined flow ducts, taking into account the presence of a boundary layer, at the high frequencies of interest associated with the modern high bypass ratio turbofan application.

Emphasis is given to the implementation of fast and efficient methods which may be used to calculate noise transmission losses at high frequencies. 'Rotor-locked' and 'rotor-stator interaction' tones generated in the fan stage, which are predominant over the broadband noise spectrum, occur at harmonics of the engine shaft frequency and blade passing frequency, respectively. In modern turbofan engines the blade 
passing frequency (BPF) corresponds to values of Helmholtz number $k^{\text {a }}$ between 20 and 30 . Therefore, the frequency range of interest goes up to values of $k$ of the order of 100, i.e. fourth or fifth harmonic of BPF. At such high frequencies, the acoustic wavelength $\lambda_{a c}$ and the boundary-layer thickness $\delta$ have comparable length scales, and the sound waves suffer refraction as a result of the non-uniformity of the flow.

In order to achieve our objectives, we restrict our attention to straight, axisymmetric (cylindrical or annular) ducts; for this simple geometry the acoustic field can be decomposed into normal-mode solutions, which yield an immediate reduction in the dimension of the problem. Following Nayfeh, Kaiser and Telionis ${ }^{2}$ and Eversman, ${ }^{3}$ each flow quantity in a turbofan engine duct can be regarded as the superposition of an unsteady, time-periodic perturbation on a steady, incompressible, inviscid, isentropic, parallel mean flow.

Let $(r, \theta, z)$ be a cylindrical polar coordinate system. At source frequency $\eta$, the acoustic field may be built up by superposition of small-amplitude, upstream- and downstream-running, shape-preserving, pressure modes of the form

$$
p(r, \theta, z, t)=\sum_{m=-\infty}^{\infty} \sum_{n=1}^{\infty}\left\{A_{m n}^{+} \psi_{m n}^{+}(r) e^{\mathrm{j}\left(\eta t-m \theta-k \lambda_{m n}^{+} z\right)}+A_{m n}^{-} \psi_{m n}^{-}(r) e^{\mathrm{j}\left(\eta t-m \theta-k \lambda_{m n}^{-} z\right)}\right\} .
$$

In equation (1) $k=\eta / c_{0}$ and $c_{0}$ is the speed of sound, the parameter $\lambda=k_{z} / k$ and $k_{z}$ is the axial wavenumber, $m \in \mathbb{Z}$ is the circumferential wavenumber, and \pm denote positive (downstream) or negative (upstream) propagating modes. For convenience, in the following we assume that $r$ and other distances have been scaled by the outer wall radius $r_{\text {out }}$, time by $r_{\text {out }} / c_{0}$, velocities by $c_{0}$, and acoustic pressure by $\rho_{r} c_{0}^{2}$ (where $\rho_{r}$ is a reference fluid density).

Assuming subsonic, shear mean flow without swirl, combining the first-order conservation equations (linearized Euler equations) and seeking solutions of the form given by equation (1), this leads to the Pridmore-Brown equation

$$
(1-M \lambda) \frac{\mathrm{d}^{2} \psi}{\mathrm{d} r^{2}}+\left[\frac{1-M \lambda}{r}+2 \lambda \frac{\mathrm{d} M}{\mathrm{~d} r}\right] \frac{\mathrm{d} \psi}{\mathrm{d} r}+(1-M \lambda)\left[k^{2}\left[(1-M \lambda)^{2}-\lambda^{2}\right]-\frac{m^{2}}{r^{2}}\right] \psi(r)=0,
$$

where $M=M(r)$ is the Mach number of the parallel mean flow. Combining equation (2) with appropriate duct wall boundary conditions leads to an eigenvalue problem to determine the eigenvalues, $\lambda$, and corresponding eigenfunctions, $\psi$. Prediction of the acoustic field at high frequencies requires that a large number of modes are calculated. Thus, it is crucial that calculations at a prescribed (high) frequency are fast and efficient.

The paper is divided into two main sections. In section II the solution of the Pridmore-Brown equation, to calculate the pressure modes at high frequencies in lined ducts with shear flow, is obtained using the finite element (FE) method. The axial wavenumbers $k_{z m n}=k \lambda_{m n}$ and the pressure mode shapes $\psi_{m n}(r)$ can be determined via the solution of a one-dimensional problem, meaning the method is very efficient even at high frequencies. Then, in section III preliminary results from the FE method are presented. Pressure modes are calculated for a number of different boundary layer profiles. Following an article in 1974 by Nayfeh, Kaiser and Shaker, ${ }^{4}$ we investigate whether the pressure modes for different boundary-layer profiles having matching thickness and/or shape factor ${ }^{\mathrm{b}}$ are similar.

\section{Finite element method}

\section{II.A. Theory}

We follow the same methodology outlined by Gabard and Astley, ${ }^{5}$ but reduce the dimension of the problem by one by taking the circumferential variation of the acoustic pressure field to vary as $e^{-\mathrm{j} m \theta}$, since we are restricting our attention to axisymmetric, circular or annular ducts. We solve the Pridmore-Brown equation (2) together with the Ingard-Myers boundary conditions

$$
\begin{array}{ll}
\frac{\mathrm{d} \psi}{\mathrm{d} r}-\mathrm{j} k A_{h}(1-M \lambda)^{2} \psi=0 & \text { on } \mathrm{r}=\mathrm{h}, \\
\frac{\mathrm{d} \psi}{\mathrm{d} r}+\mathrm{j} k A_{1}(1-M \lambda)^{2} \psi=0 & \text { on } \mathrm{r}=1,
\end{array}
$$

${ }^{a}$ Non-dimensional free field wavenumber scaled by a suitable lengthscale, which in this context is the outer wall radius of the duct, $r_{\text {out }}$.

${ }^{\mathrm{b}}$ The shape factor is the ratio of displacement to momentum thickness. 
where $A_{h}$ and $A_{1}$ denote the non-dimensional specific acoustic admittance of the inner and outer duct wall, respectively. ${ }^{\mathrm{c}}$ The problem is recast, after some lengthy algebraic manipulations, as the following variational formulation

$$
\alpha+\lambda \beta+\lambda^{2} \gamma+\lambda^{3} \delta=0
$$

where

$$
\begin{aligned}
\alpha= & -\int_{h}^{1}\left(\frac{\mathrm{d} \phi}{\mathrm{d} r} \frac{\mathrm{d} \psi}{\mathrm{d} r}+\frac{m^{2}}{r^{2}} \phi \psi\right) r \mathrm{~d} r+k^{2} \int_{h}^{1} \phi \psi r \mathrm{~d} r-\left(\left.A \phi \psi r\right|_{r=1}+\left.A \phi \psi r\right|_{r=h}\right), \\
\beta= & \int_{h}^{1} M\left(\frac{\mathrm{d} \phi}{\mathrm{d} r} \frac{\mathrm{d} \psi}{\mathrm{d} r}+\frac{m^{2}}{r^{2}} \phi \psi\right) r \mathrm{~d} r-3 k^{2} \int_{h}^{1} M \phi \psi r \mathrm{~d} r \\
& +3 \mathrm{j} k\left(\left.A M \phi \psi r\right|_{r=1}+\left.A M \phi \psi r\right|_{r=h}\right)+3 \int_{h}^{1} \phi \frac{\mathrm{d} M}{\mathrm{~d} r} \frac{\mathrm{d} \psi}{\mathrm{d} r} r \mathrm{~d} r, \\
\gamma= & k^{2}\left(3 \int_{h}^{1} M^{2} \phi \psi r \mathrm{~d} r-\int_{h}^{1} \phi \psi r \mathrm{~d} r\right)-3 \mathrm{j} k\left(\left.A M^{2} \phi \psi r\right|_{r=1}+\left.A M^{2} \phi \psi r\right|_{r=h}\right), \\
\delta= & -k^{2}\left(\int_{h}^{1} M^{3} \phi \psi r \mathrm{~d} r-\int_{h}^{1} M \phi \psi r \mathrm{~d} r\right)+\mathrm{j} k\left(\left.A M^{3} \phi \psi r\right|_{r=1}+\left.A M^{3} \phi \psi r\right|_{r=h}\right),
\end{aligned}
$$

and $\phi$ is any sufficiently regular function.

Subdividing the solution domain, $h \leq r \leq 1$, into $N_{E}$ finite elements, we replace $\psi$ and $\phi$ by their approximations of the form

$$
\psi(r)=\sum_{i=1}^{N_{G}} \psi_{i} N_{i}(r) \quad \text { and } \quad \phi(r)=\sum_{i=1}^{N_{G}} \phi_{i} N_{i}(r),
$$

where $\left\{N_{i}\right\}_{i=1,2, \ldots N_{G}}$ is a finite element basis, $N_{G}$ is the number of global nodes (which depend on the degree of the Lagrangian element selected), and the coefficients $\psi_{i}$ are the unknown values at the nodes $\psi_{i}=\psi\left(r_{i}\right)$. A cubic eigenvalue problem for $\lambda$ is obtained, expressed compactly in the form

$$
\mathbf{C}(\lambda) \psi=0 .
$$

The matrix $\mathbf{C}(\lambda)$ is an $N_{G}$ by $N_{G}$ matrix polynomial defined as

$$
\mathbf{C}(\lambda)=\sum_{n=0}^{3} \lambda^{n} \mathbf{C}_{n}
$$

and $\psi$ is the vector defined by

$$
\boldsymbol{\psi}^{T}=\left[\psi_{1}, \psi_{2}, \ldots \psi_{N_{G}}\right] .
$$

$\mathbf{C}_{n}$ are defined as

$$
\begin{aligned}
& \mathbf{C}_{0}=k^{2} \mathbf{M}^{(0)}-\mathbf{K}^{(0)}-\mathrm{j} k \mathbf{Z}^{(0)}, \\
& \mathbf{C}_{1}=-3 k^{2} \mathbf{M}^{(1)}+\mathbf{K}^{(1)}+3 \mathrm{j} k \mathbf{Z}^{(1)}+3 \mathbf{S}, \\
& \mathbf{C}_{2}=k^{2}\left(3 \mathbf{M}^{(2)}-\mathbf{M}^{(0)}\right)-3 \mathrm{j} k \mathbf{Z}^{(2)}, \\
& \mathbf{C}_{3}=-k^{2}\left(\mathbf{M}^{(3)}-\mathbf{M}^{(1)}\right)+\mathrm{j} k \mathbf{Z}^{(3)},
\end{aligned}
$$

\footnotetext{
${ }^{\mathrm{c}}$ The non-dimensional specific acoustic impedance $Z=1 / A$.
} 
where $\mathbf{M}^{(n)}, \mathbf{K}^{(n)}, \mathbf{Z}^{(n)}$ and $\mathbf{S}$, for $n=0,1,2,3$, are defined, in index notation, as

$$
\begin{aligned}
M_{i j}^{(n)} & =\int_{h}^{1}[M(r)]^{n} N_{i} N_{j} r \mathrm{~d} r, \\
K_{i j}^{(n)} & =\int_{h}^{1}[M(r)]^{n}\left(\frac{\mathrm{d} N_{i}}{\mathrm{~d} r} \frac{\mathrm{d} N_{j}}{\mathrm{~d} r}+\frac{m^{2}}{r^{2}} N_{i} N_{j}\right) r \mathrm{~d} r, \\
Z_{i j}^{(n)} & =\left.A_{1}[M(r)]^{n} N_{i} N_{j} r\right|_{r=1}+\left.A_{h}[M(r)]^{n} N_{i} N_{j} r\right|_{r=h}, \\
S_{i j} & =\int_{h}^{1} N_{j} \frac{\mathrm{d} M}{\mathrm{~d} r} \frac{\mathrm{d} N_{i}}{\mathrm{~d} r} r \mathrm{~d} r .
\end{aligned}
$$

Equation (6) can be expressed as a linear eigenvalue problem of the form

$$
\mathbf{A q}=\lambda \mathbf{B q}
$$

where

$$
\mathbf{A}=\left[\begin{array}{ccc}
\mathbf{0} & \mathbf{I} & \mathbf{0} \\
\mathbf{0} & \mathbf{0} & \mathbf{I} \\
-\mathbf{C}_{0} & -\mathbf{C}_{1} & -\mathbf{C}_{2}
\end{array}\right], \quad \mathbf{B}=\left[\begin{array}{ccc}
\mathbf{I} & \mathbf{0} & \mathbf{0} \\
\mathbf{0} & \mathbf{I} & \mathbf{0} \\
\mathbf{0} & \mathbf{0} & \mathbf{C}_{3}
\end{array}\right], \quad \mathbf{q}=\left[\begin{array}{c}
\boldsymbol{\psi} \\
\lambda \boldsymbol{\psi} \\
\lambda^{2} \boldsymbol{\psi}
\end{array}\right]
$$

and $\mathbf{0}$ and $\mathbf{I}$ are the null and identity matrices, respectively. The matrices $\mathbf{C}_{n}$ are obtained via the usual finite element assembly process.

\section{II.B. Implementation}

The FE method has traditionally been coded in procedural programming paradigm. However, since the introduction of the object oriented paradigm in the early 1990s, a number of articles on applying this programming style to the FE method have been published. Some of the first papers on the subject are by Zimmermann, Dubois-Pelerin and Bomme ${ }^{6}$ and Mackie, ${ }^{7}$ both published in 1992. In our work the object-oriented programming style has been adopted for the development of the FE code. Details of the implementation are not included here. However, the supported configurations which are included in the code are listed below.

1. Field equation: convected Helmholtz equation or Pridmore-Brown equation.

2. Boundary conditions: rigid or acoustically-lined (locally-reacting) duct wall.

3. Duct geometry: axisymmetric, circular or annular waveguide.

4. Mean flow: parallel shear flow, Mach number $M(r)$.

5. FE implementation: linear and quadratic Lagrange basis functions; numeric and symbolic (where possible) integration schemes; solution of the generalized eigenvalue problem (11) via the QZ-algorithm due to Moler and Stewart ${ }^{8}$ from the IMSL C library.

6. Post-processing: routine to identify and sort the modes (i.e. hydrodynamic or acoustic, positive or negative propagating modes).

\section{II.C. Validation}

The purpose of this subsection is to quantify the accuracy of the numerical model by inspecting the convergence of the relative error of the axial wavenumbers, by increasing the number of mesh points. The relative error used here is defined as

$$
e=\frac{\left|k_{z}-k_{z, \mathrm{ref}}\right|}{\left|k_{z, \mathrm{ref}}\right|}
$$


where $k_{z, r e f}$ is a reference axial wavenumber obtained numerically with a very high-resolution mesh.

The mesh resolution is typically characterized by the number of elements per wavelength

$$
n_{e}=\frac{\lambda_{a c}}{h}
$$

where $\lambda_{a c}$ is the acoustic wavelength and $h$ is the typical element size in a given mesh. Therefore we define $k h=2 \pi / n_{e}$, where $k$ is the Helmholtz number and $h$ is the non-dimensional element size scaled by the outer duct radius $r_{\text {out }}$.

The test case of a lined annular duct with hub-to-tip ratio $h=0.8$, uniform mean flow $M_{0}=0.5$, nondimensional specific acoustic impedance $Z=2-3 \mathrm{j}$, and Helmholtz number $k=20$ is considered. The relative error, $e$, is plotted as a function of the radial mode order and for different mesh resolutions in figure 1. The resolution of the mesh used to produce $k_{z \text {,ref }}$ is $k h=0.01142$. As a general trend, the relative error grows as modes of higher radial order are considered. This is expected as the higher the radial order, the resolution of the mode becomes less accurate using the finite element method. For the cut-on modes, results obtained with relatively coarse meshes are very similar to those obtained with much finer meshes. Moreover, the relative error reduces systematically for all the modes shown here when using finer meshes. Also the relative error as a function of $k h$ for a number of prescribed modes is shown plotted in figure 2. Again this shows how, in general, the relative error falls as $k h$ is reduced.

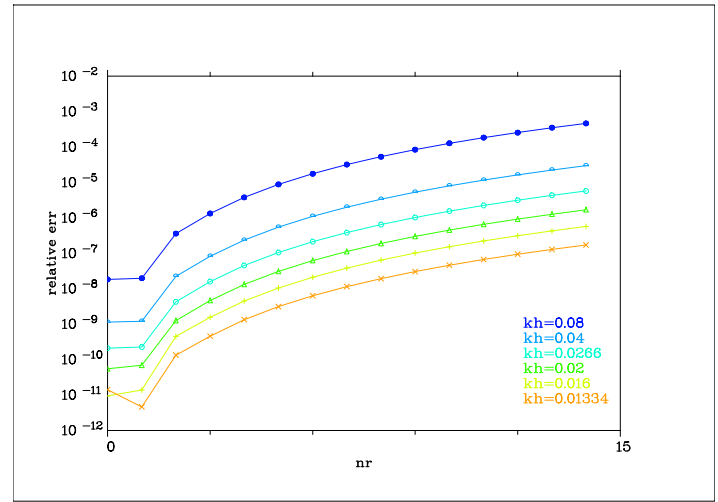

(a)

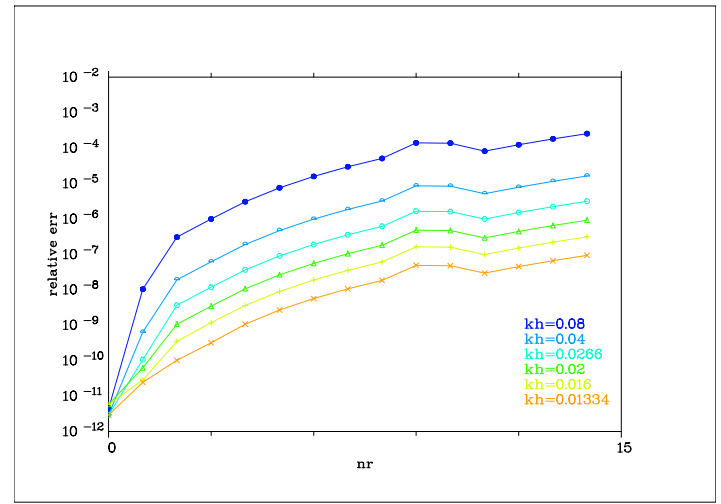

(b)

Figure 1. Relative error of the calculated axial wavenumbers with different mesh resolutions for the test case of a lined annular duct with uniform flow, $h=0.8, M_{0}=0.5, Z=2-3 \mathrm{j}, k=20$ : (a) $m=0$, negative (upstream) modes, (b) $m=0$, positive (downstream) modes.

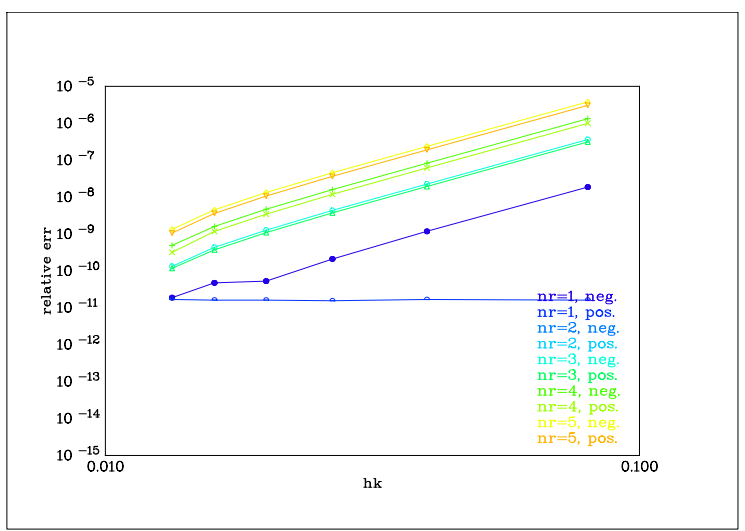

Figure 2. Convergence of the calculated axial wavenumbers with increased mesh resolution for the test case of a lined annular duct with uniform flow, $h=0.8, M_{0}=0.5, Z=2-3 \mathrm{j}, k=20$. Convergence is shown for positive and negative modes, radial orders $n=1$ to 5 . 


\section{Preliminary results}

Preliminary results are shown for both a rigid and an acoustically-lined annular duct. In the case of the lined duct, results are provided for a locally-reacting acoustic lining with (non-dimensional) specific acoustic impedance $Z=2-3 \mathrm{j}$. Sample results are provided for an annular duct with hub-to-tip ratio $h=0.8$, and a frequency which corresponds to Helmholtz number $k=20$.

The eigenmodes have been computed for three different mean-flow profiles:

1. Uniform flow;

2. Linear boundary-layer;

3. $1 / 7$ th power law boundary-layer.

The flow profiles for the shear flow test cases are

$$
M(r)= \begin{cases}M_{0}\left(\frac{r-r_{\text {in }}}{\delta}\right)^{1 / N} & r_{\text {in }} \leqslant r<r_{\text {in }}+\delta, \\ M_{0} & r_{\text {in }}+\delta \leqslant r \leqslant r_{\text {out }}-\delta \\ M_{0}\left(\frac{r_{\text {out }}-r}{\delta}\right)^{1 / N} & r_{\text {out }}-\delta<r \leqslant r_{\text {out }}\end{cases}
$$

where $M_{0}$ is the core flow Mach number, $r_{\text {out }}$ and $r_{\text {in }}$ are the outer and inner wall duct radius, and $N$ defines the power $(N=1$ for case (2), $N=7$ for case (3)). Also, in all the results the uniform flow is taken to be $M_{0}=0.5$.

Our objective is to examine the pressure modes for different boundary-layer profiles. For each test case, the eigenvalues $\lambda=k_{z} / k$ are shown plotted on an Argand diagram. Only the acoustic modes are shown (the continuous spectrum of hydrodynamic modes is not shown). Both positive (downstream) and negative (upstream) running modes are shown. The linear boundary layer profile can be adjusted by permitting a slip velocity at the wall. ${ }^{\mathrm{d}}$ Varying the velocity at the duct wall allows the shape factor of the profile to be altered, whilst keeping the boundary-layer thickness fixed. Accordingly, in all the following test cases, each boundarylayer profile has the same thickness $\delta$, but two linear boundary-layer profiles are assessed. One profile has no slip at the wall, whilst the other profile has a slip velocity (duct wall Mach number $0 \leq M_{s} \leq M_{0}$ ) such that the linear with slip boundary-layer profile has the same shape factor as the $1 / 7$ th power law boundary layer. This allows comparisons to be made between two different profiles having matching thickness and/or matching shape factor as well. Results are provided taking the non-dimensional boundary layer thickness to be $\delta=0.01\left(1 \%\right.$ of $\left.r_{\text {out }}\right)$ and $\delta=0.05\left(5 \%\right.$ of $\left.r_{\text {out }}\right)$.

Figures 3 and 4 show the eigenvalues $\lambda=k_{z} / k$ for a rigid and a lined annular duct with $k=20$, respectively. Modes having circumferential order $m=0$ and $m=10$ are shown, for boundary-layer profiles with thickness $\delta=0.01$ and $\delta=0.05$. Some examples of the pressure mode shapes $\psi(r)$ are shown in figures 5 and 6 . These show the positive and negative modes with circumferential order $m=0$, and radial orders $n=1$ and 2. The boundary layer thickness is $\delta=0.05$. Finally, in figure 7 a further example of the eigenvalues $\lambda=k_{z} / k$ is provided for a rigid annular duct with $k=20$ and $\delta=0.05$, now showing a range of circumferential orders from $m=0$ to $m=20$.

\section{Discussion}

Whilst no definitive conclusions are possible based on the small selection of preliminary results presented in section III, there are several features which can be observed in these results which warrant further investigation. In figures 3 and 4 it is seen that the inclusion of a boundary layer alters the eigenvalues (axial wavenumbers $k_{z}=k \lambda$ ), and unsurprisingly there is greater variation with the thicker boundary-layer profile. There are no clear trends evident when comparing the eigenvalues for the different profiles, albeit there is slightly more variation in the values of $\lambda$ with the negative propagating modes, i.e. modes propagating against the mean flow. Conversely, in the rigid duct, the values of $\lambda$ for the positive propagating (cut-on) modes are very similar for each of the different boundary-layer profiles.

\footnotetext{
${ }^{\mathrm{d}}$ This requires a slight modification to equation (13) to allow a non-zero Mach number at $r=r_{\text {in }}$ and/or $r=r_{\text {out }}$.
} 
The other notable feature in these results is that with the $1 / 7$ th power law boundary layer and $\delta=0.05$, there are two isolated modes in the upper and lower right quadrants of the complex $\lambda$-plane, which have shifted from the upper and lower left quadrants where all the other modes in their families are located. This does not occur with the linear boundary layer. These eigenvalues are seen in both figures 3 and 4 with the rigid and lined duct, and their values are similar in both cases. Accordingly, these modes are not related to the presence of the acoustic liner, such as surface acoustic waves which can be detected in lined ducts. Inspection of the pressure mode shapes has confirmed that these are, also, acoustic modes.

In figures 5 and 6 there are comparisons of some low-order pressure mode shapes (eigenfunctions $\psi(r)$ ) with circumferential order $m=0$. As expected the largest variation in the mode shapes occur for $n=1$. In the rigid duct, $(m, n)=(0,1)$ is the plane wave mode. The inclusion of a boundary layer means that this mode shape is not planar, hence the seemingly large variation in the eigenfunctions for this mode order. There is less variability in radial order $n=2$, although as already noted the variability is greater with the negative propagating modes. Although these results are only for a limited number of test cases, it is seen that for these low-order modes the 1/7th power law boundary layer and the linear without slip boundary layer have similar mode shapes for this test case.

In figure 7 there is a further example of the eigenvalues $\lambda$ with Helmholtz number equal to 20, for a range of azimuthal orders from $m=0$ to 20 . The families of modes calculated with a boundary layer are markedly different to the modes for uniform flow. Comparing the eigenvalues for the $1 / 7$ th power law and linear (without slip) boundary layers, these appear similar apart from the presence of clusters of modes in the upper and lower right quadrants with the power law profile. These clusters of modes appear in the upper and lower left quadrants with the linear profile, so appear to have been shifted to the right hand side of the $\lambda$-plane with the power law profile.

\section{Conclusions}

The objective of this work is the development of fast and efficient methods to calculate the pressure modes in lined flow ducts at high frequencies. In the case of axisymmetric, circular or annular ducts, a finite element method has been found to provide an efficient calculation method at high frequencies, because only a one-dimensional mesh is required since the pressure mode shapes only depend on radial position. Thus, calculations at very high frequencies are possible (with only modest computational cost), providing large quantities of modes per simulation. Typically around 300 elements have been used to produce the results shown in this paper.

Having calculated a set of modes for a prescribed test case, part of the post-processing requires that these modes are classified and sorted. This is not a trivial task, but it is essential since there will be different types of solutions calculated by the FE method. This includes modes which are predominantly acoustic or hydrodynamic, as well as positive and negative propagating modes. We are currently evaluating a procedure which determines the group velocity of the modes, in order to verify their propagation direction, and also that the modes are stable.

A number of previous articles have examined the pressure modes in a duct with thin boundary layers on the walls. With a thin boundary layer, it may be reasonable to neglect the boundary layer and assume that the mean flow is uniform. It has also been postulated that with a thin boundary layer, the effect of the profile shape is not particularly significant on the attenuation of the low-order modes in a lined duct. We have started to examine a linear boundary layer, to assess whether this profile could be used in place of other, more realistic, boundary-layer profiles when calculating the pressure modes. This is an attractive profile to consider since it offers the potential to find analytic solutions, for example, see Goldstein and Rice. ${ }^{9}$ However, some caution must be exercised because the profile's gradient has a discontinuity at the edge of the boundary layer.

Our future aim is that approximate solutions for boundary-layer profiles (calculated using perturbation methods) will be compared to full numerical solutions calculated using the finite element method, at high sound frequencies. The validity of using approximation methods at high frequencies will be investigated. The computational effort using approximation methods will be similar to that required to compute the pressure modes at high frequencies with uniform mean flow (for which a closed-form analytic solution exists). This offers an attractive approach whereby the effect of duct wall boundary layers can be included in the modelling without significantly increasing the complexity or cost of the modelling procedure. 


\section{Acknowledgments}

The work reported in this article was supported by the Engineering and Physical Sciences Research Council (EPSRC) and Rolls-Royce plc through the Rolls-Royce University Technology Centre in Gas Turbine Noise at the University of Southampton.

\section{References}

\footnotetext{
${ }^{1}$ M.K. MYERS. On the acoustic boundary condition in the presence of flow. Journal of Sound and Vibration, 71(3):429-434, 1980.

${ }^{2}$ A.H. NAYFEH, J.E. KAISER, and D.P. TELIONIS. Acoustics of aircraft engine-duct systems. AIAA Journal, 13(2):130$153,1975$.

${ }^{3}$ W. EVERSMAN. Theoretical models for duct acoustic propagation and radiation. In Aeroacoustics of flight vehicles: theory and practice vol. 2 Noise control, pages 101-163. NASA RP-1258, 1991.

${ }^{4}$ A.H. NAYFEH, J.E. KAISER, and B.S. SHAKER. Effect of mean-velocity profile shapes on sound transmission through two-dimensional ducts. Journal of Sound and Vibration, 34:413-423, 1974.

${ }^{5}$ G. GABARD and R.J. ASTLEY. A computational mode-matching approach for sound propagation in three-dimensional ducts with flow. Journal of Sound and Vibration, 315:1103-1124, 2008.

${ }^{6}$ T. ZIMMERMANN, Y. DUBOIS-PELERIN, and P. BOMME. Object-oriented finite-element programming. 1. Governing principles. Computer Methods in Applied Mechanics and Engineering, 98(2):291-303, 1992.

${ }^{7}$ R.I. MACKIE. Object oriented programming of the finite-element method. International Journal for Numerical Methods in Engineering, 35(2):425-436, 1992.

${ }^{8}$ C.B. MOLER and G.W. STEWART. Algorithm for generalized matrix eigenvalue problems. SIAM Journal on Numerical Analysis, 10(2):241-256, 1973.

${ }^{9}$ M. GOLDSTEIN and E. RICE. Effect of shear on duct wall impedance. Journal of Sound and Vibration, 30(1):79-84, 1973.
} 


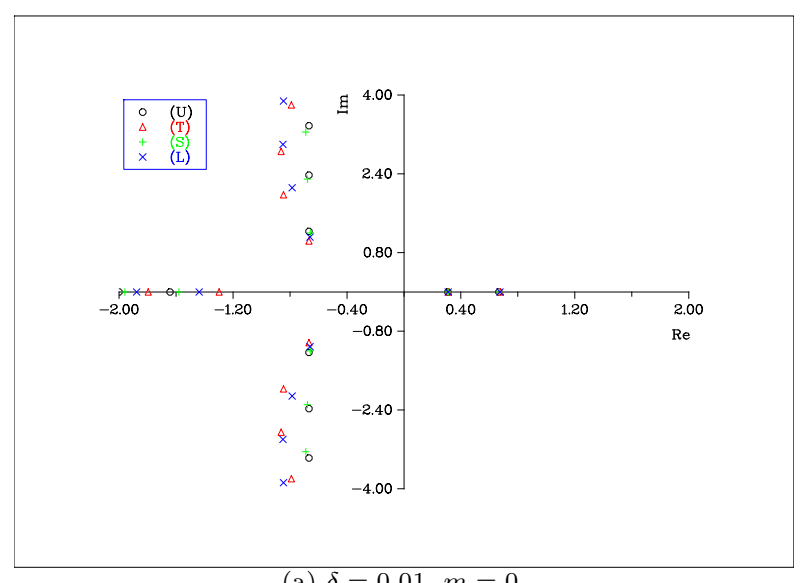

(a) $\delta=0.01, m=0$.

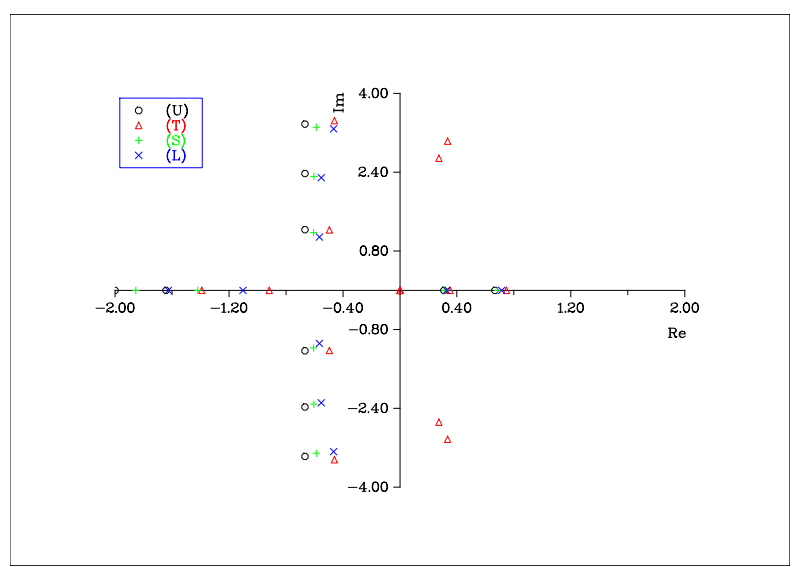

(c) $\delta=0.05, m=0$.

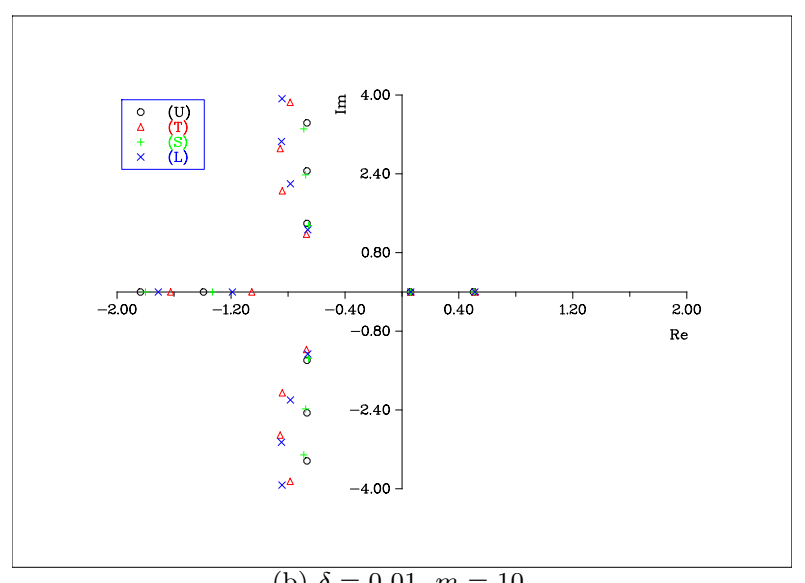

(b) $\delta=0.01, m=10$.

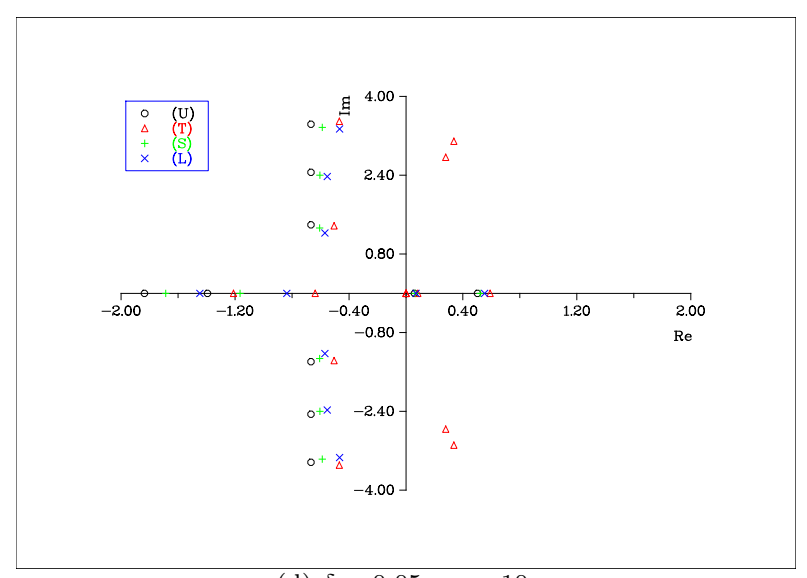

(d) $\delta=0.05, m=10$.

Figure 3. Eigenvalues $\lambda=k_{z} / k$ for a rigid annular duct $\left(h=0.8, M_{0}=0.5, k=20\right)$ with mean flow profiles: (U) Uniform flow; (T) 1/7th power law boundary-layer; $(S)$ Linear boundary-layer with slip; $(L)$ Linear boundary-layer without slip. 


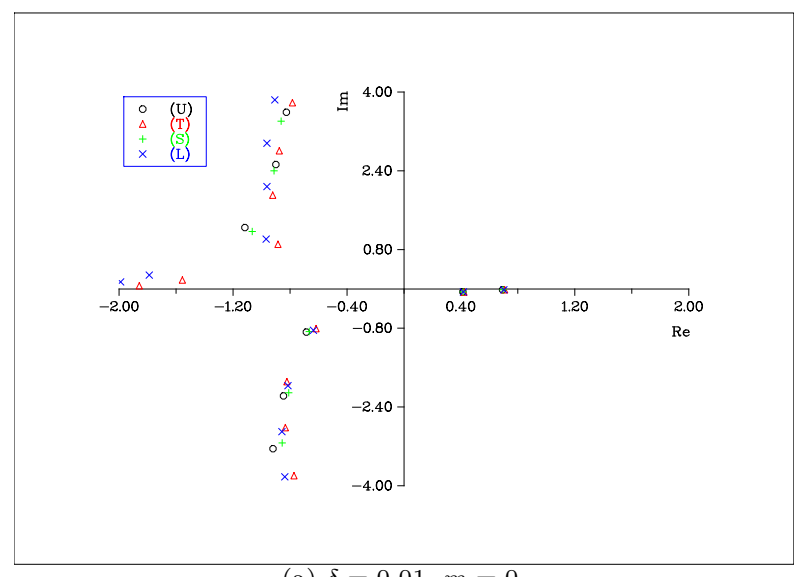

(a) $\delta=0.01, m=0$.

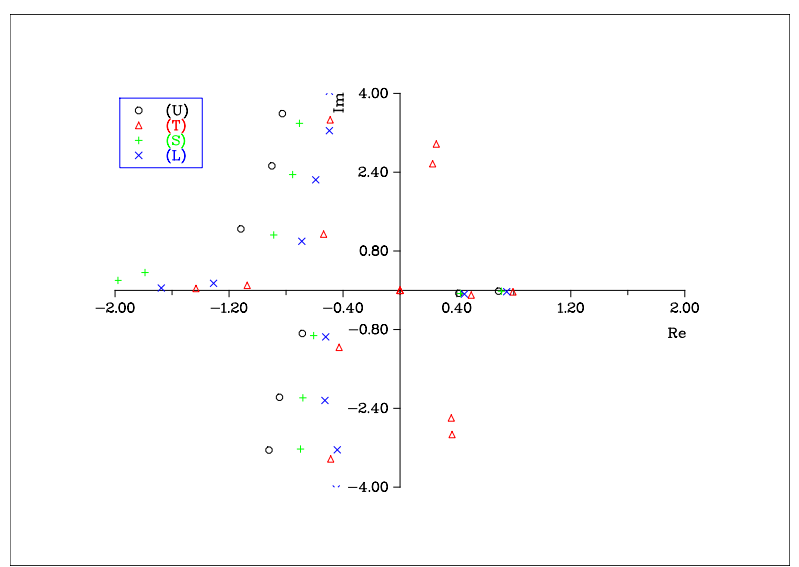

(c) $\delta=0.05, m=0$.

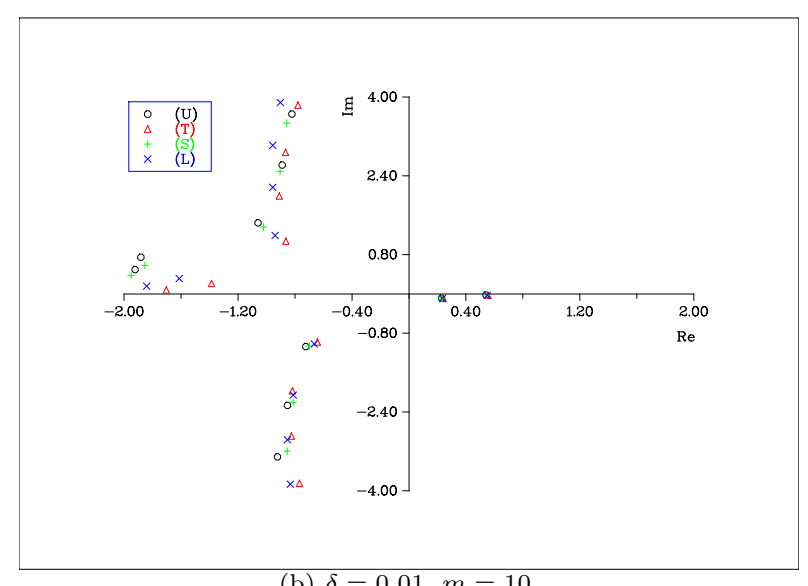

(b) $\delta=0.01, m=10$.

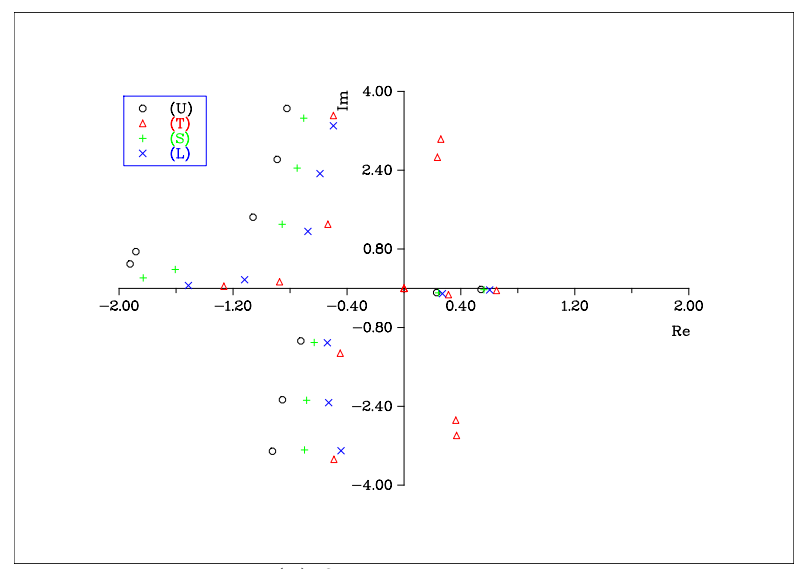

(d) $\delta=0.05, m=10$.

Figure 4. Eigenvalues $\lambda=k_{z} / k$ for a lined annular duct $\left(h=0.8, M_{0}=0.5, k=20, Z=2-3 \mathrm{j}\right)$ with mean flow profiles: $(U)$ Uniform flow; $(T)$ 1/7th power law boundary-layer; $(S)$ Linear boundary-layer with slip; $(L)$ Linear boundary-layer without slip. 


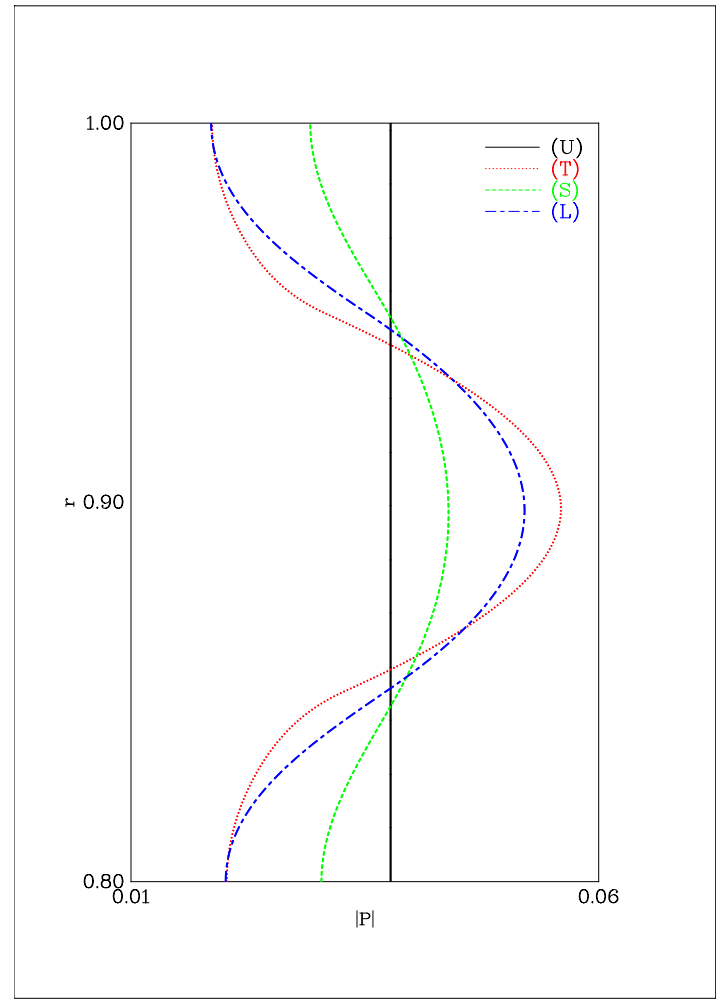

(a) Negative (upstream) modes; $n=1$

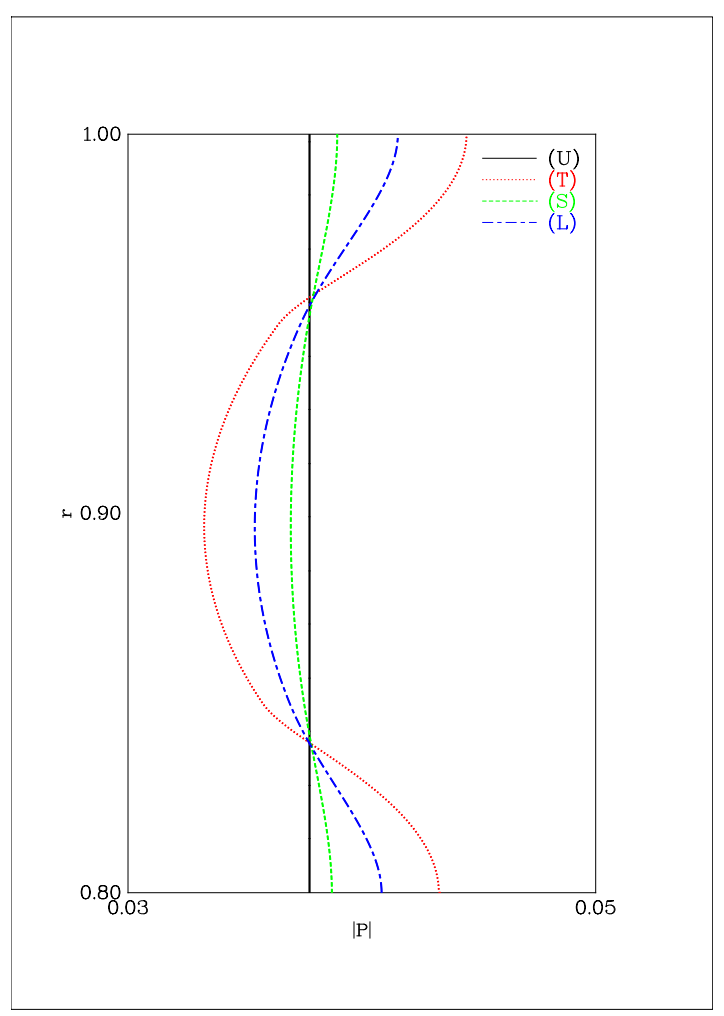

(c) Positive (downstream) modes; $n=1$

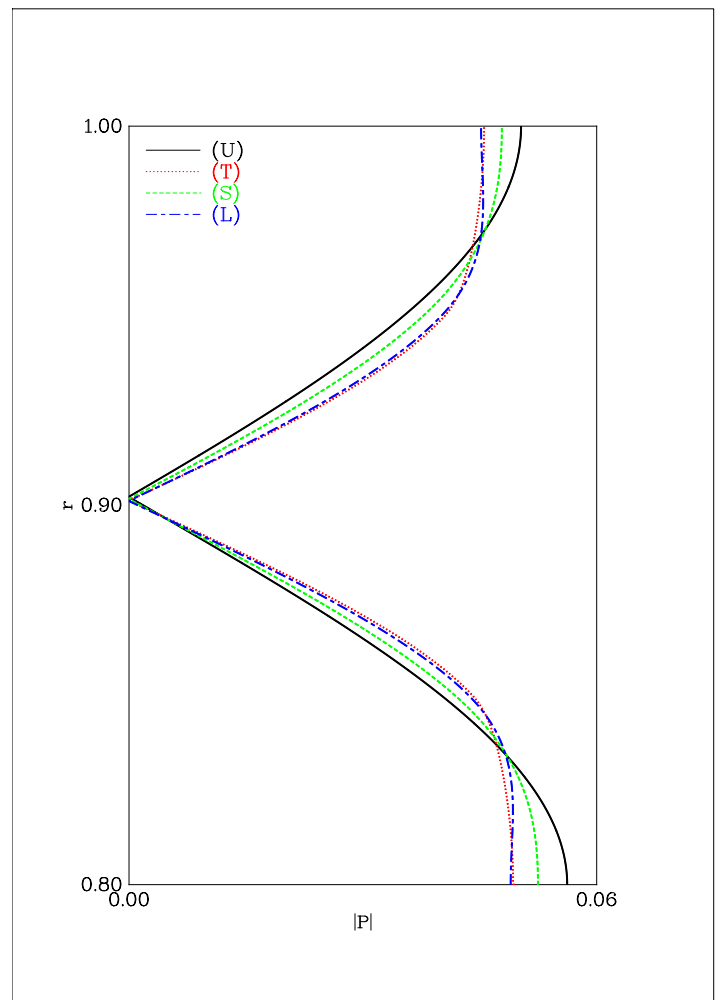

(b) Negative (upstream) modes; $n=2$

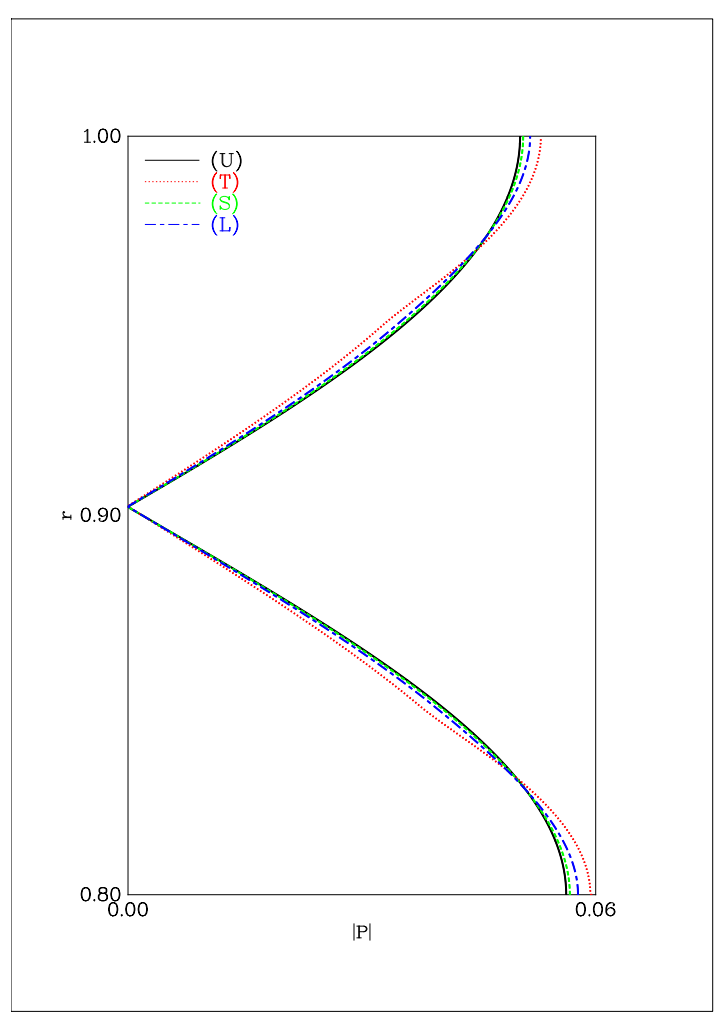

(d) Positive (downstream) modes; $n=2$

Figure 5. Examples of pressure mode shapes $\psi(r)$ for a rigid annular duct $\left(h=0.8, M_{0}=0.5, \delta=0.05, k=20\right)$ for circumferential order $m=0$. Mode shapes are shown for different flow profiles-see key. 


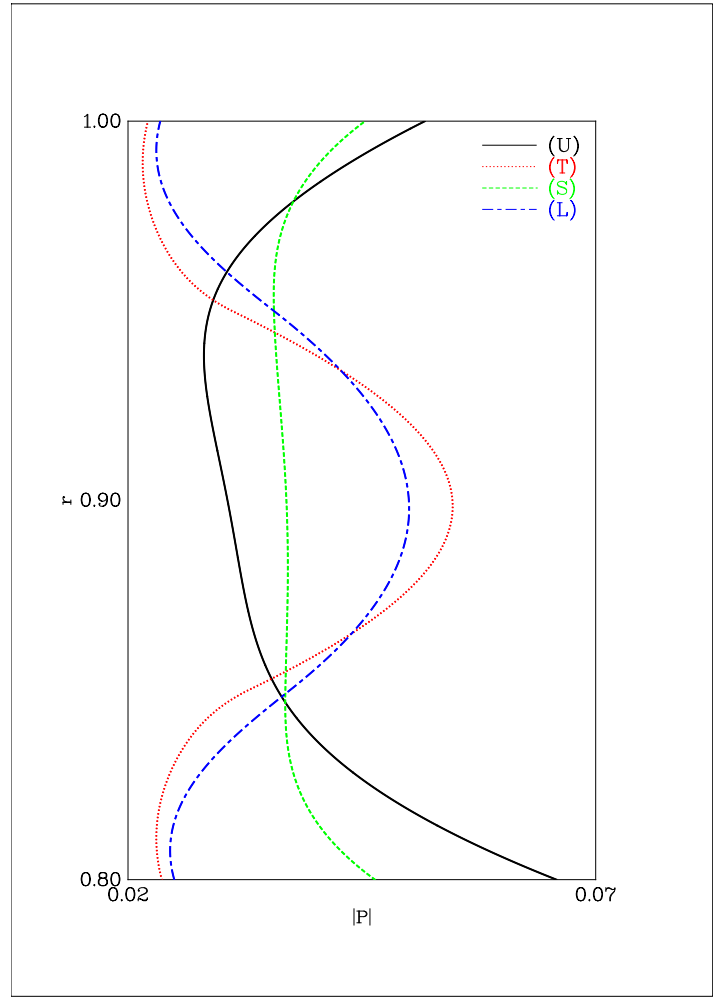

(a) Negative (upstream) modes; $n=1$

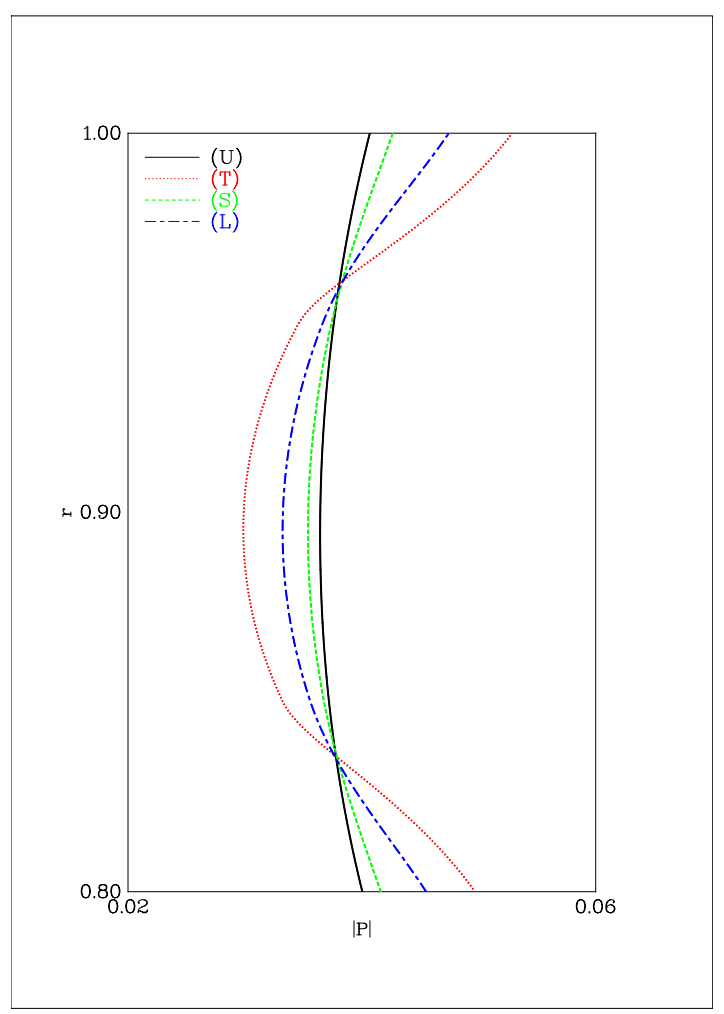

(c) Positive (downstream) modes; $n=1$

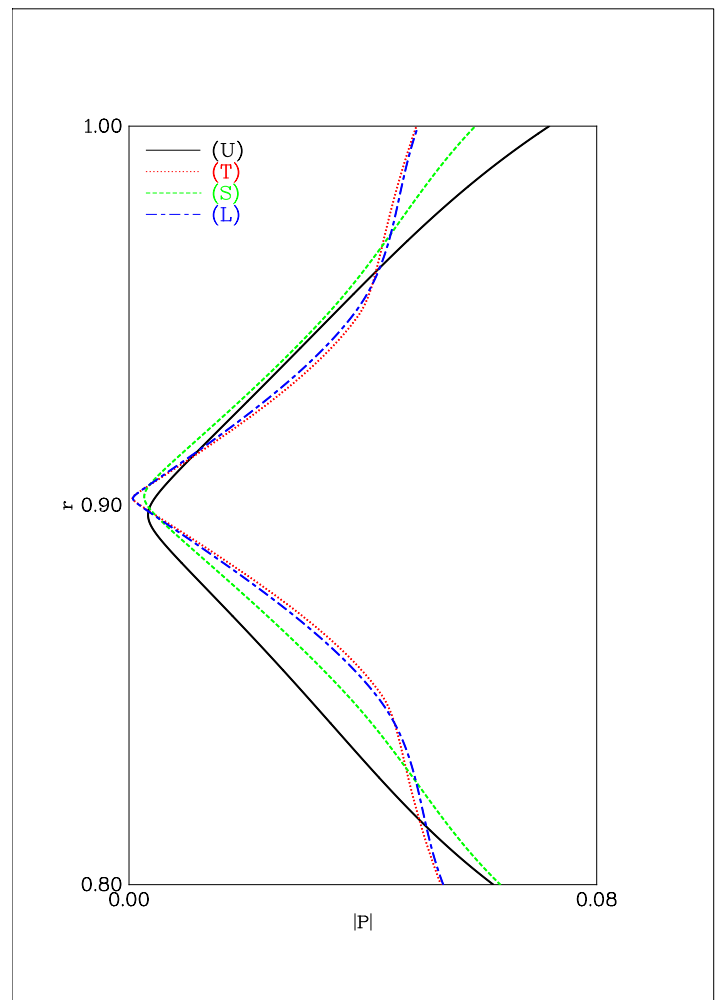

(b) Negative (upstream) modes; $n=2$

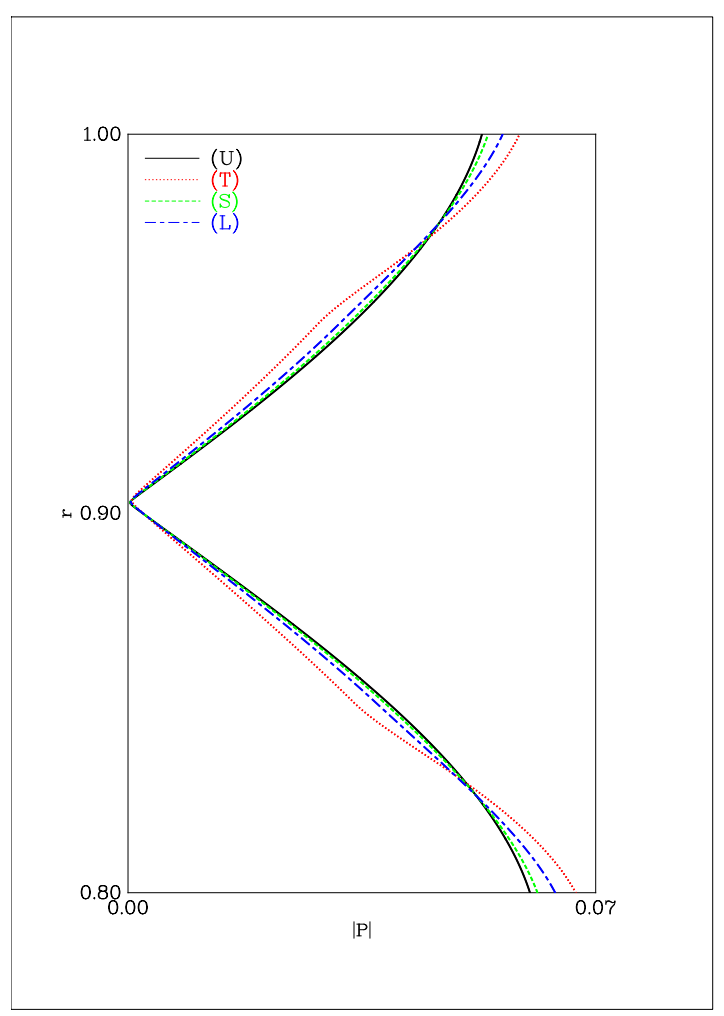

(d) Positive (downstream) modes; $n=2$

Figure 6. Examples of pressure mode shapes $\psi(r)$ for a lined annular duct $\left(h=0.8, M_{0}=0.5, \delta=0.05, k=20, Z=2-3 \mathrm{j}\right)$ for circumferential order $m=0$. Mode shapes are shown for different flow profiles-see key. 


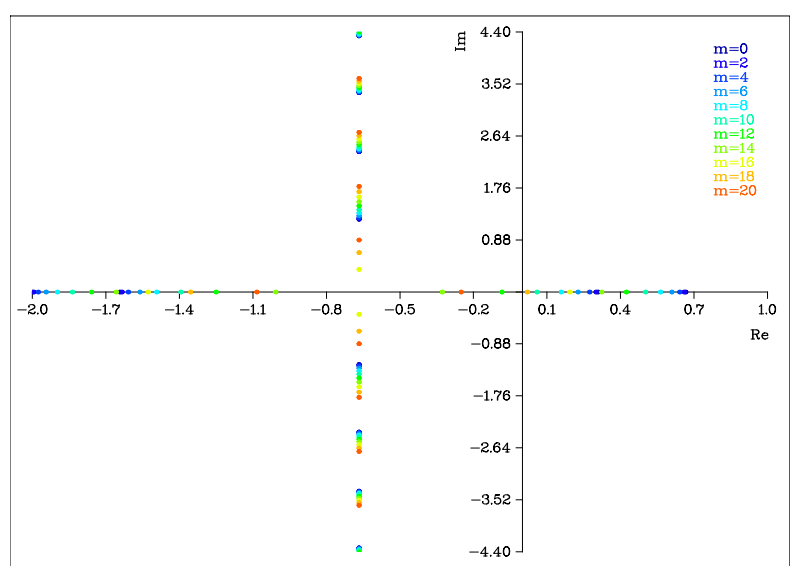

(a) Uniform flow.

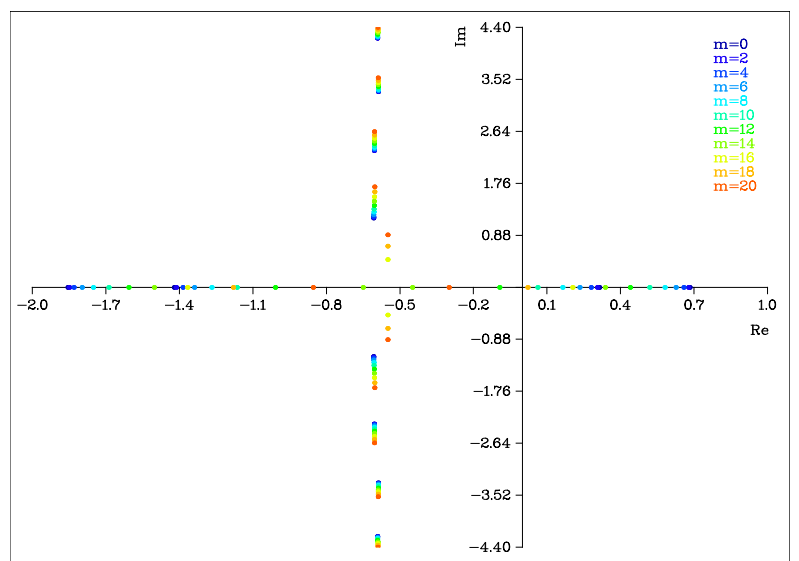

(c) Linear with slip boundary layer, $\delta=0.05$.

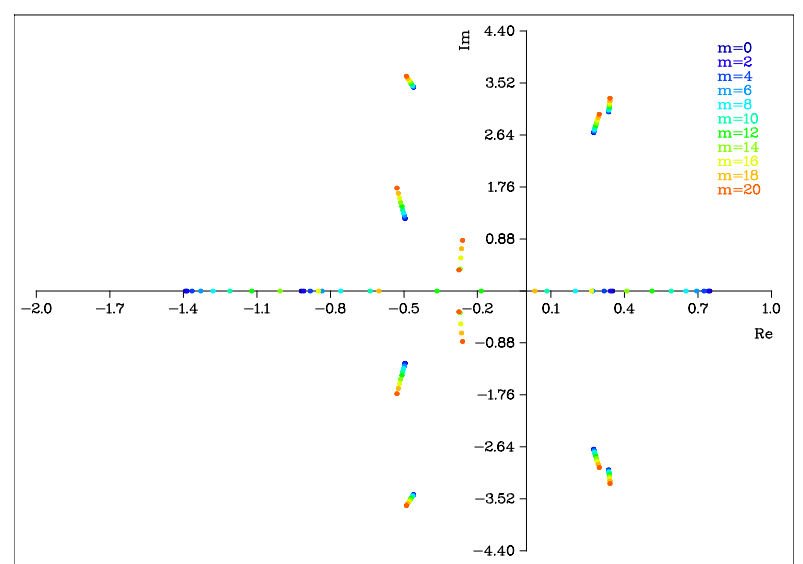

(b) $1 / 7$ th power law boundary layer, $\delta=0.05$.

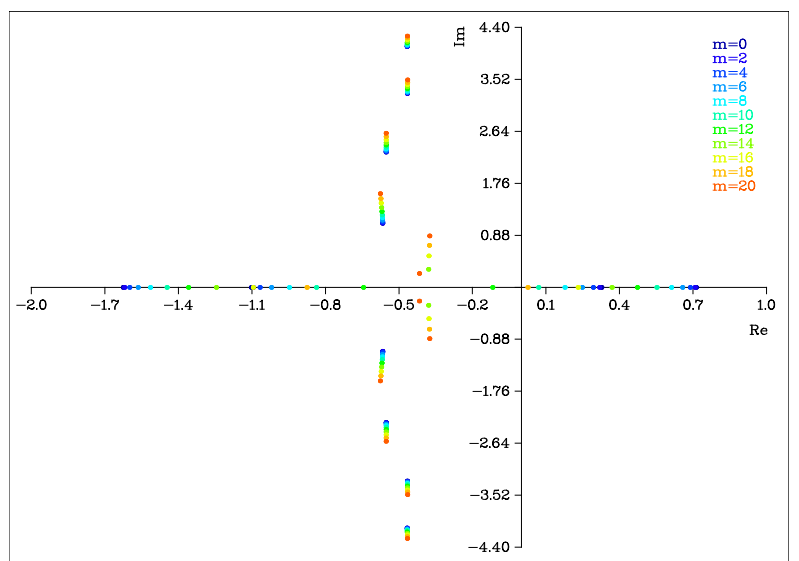

(d) Linear without slip boundary layer, $\delta=0.05$.

Figure 7. Eigenvalues $\lambda=k_{z} / k$ for a rigid annular duct $\left(h=0.8, M_{0}=0.5, k=20\right)$. 